\title{
Fastest-Path Planning for Direction-Dependent Speed Functions
}

\author{
Irina S. Dolinskaya * $\quad$ Robert L. Smith ${ }^{\dagger}$
}

\begin{abstract}
We discuss path planning in a direction-dependent environment illustrated by the fastestpath problem with anisotropic speed function. The difficulty of optimal-path finding in a direction-dependent medium comes from the fact that our travel-time function is asymmetric, and in general, violates the triangle inequality. We present an analytical form solution for the fastest-path finding problem in an obstacle-free domain without making any assumptions on the structure of the speed function. Subsequently, we merge these results with visibility graph search methods to develop an obstacle-avoiding fastest-path finding algorithm for an anisotropic speed function. Optimal routing of a vessel in a stationary random seaway is discussed throughout the paper to motivate and demonstrate application of our work.
\end{abstract}

\section{Introduction}

In this paper, we address a broad class of optimal path finding in anisotropic environment problems where the cost function is direction-dependent. For ease of exposition, we focus our discussion on fastest-path finding problems for direction-dependent speed functions; however, our analysis and results can be easily extended to any anisotropic cost function. We are given the points of origin and destination, and time and space homogenous speed function of heading. Our objective is to find a path that minimizes the total travel time. Problems of optimal path finding in an obstacle-free domain, as well as in the presence of polygonal obstacles, are addressed.

One of the applications for this type of problem is optimal short-range routing of vessels in a seaway, which motivated this research. In the recent years, comprehensive seakeeping models have been developed to accurately estimate the effects of waves on vessel performance. In [1], the average added drag of the head and oblique waves is evaluated in a stationary random seaway. In addition, a number of operability constraints, such as probability of wet deck and root-mean-squared roll values, are computed for various vessel headings and sea states (i.e., wave-field distributions). The integrated seakeeping model delivers the expected maximum attainable speed for a containership as a function of its heading angle relative to the dominant wave direction. The resulting anisotropic speed function has irregular structure that can not be evaluated analytically. Our analysis makes no assumptions about the structure of the direction-dependent cost function and can be applied to the very general set of problems, including the vessel routing problem presented here. Furthermore, integration of obstacles into the path domain allows one to incorporate restricted sea regions, presence of shallow waters, land and other vessels into the path planning process.

The difficulty of optimal-path finding in an anisotropic medium comes from the fact that our travel-time function is asymmetric; that is, the time it takes to travel along a straight line path

\footnotetext{
${ }^{*}$ Department of Industrial Engineering and Management Sciences, Northwestern University, Evanston, IL 60208, dolira@northwestern.edu

${ }^{\dagger}$ Department of Industrial and Operations Engineering, University of Michigan, Ann Arbor, Michigan 48109, rlsmith@umich.edu
} 
from $a$ to $b$ does not necessarily equal the time required to traverse the reversed path $b a$. Therefore, our cost function is not a metric, which prevents us from using more traditional and established approaches to solving optimal-path finding problems. Furthermore, the anisotropic cost, in general, violates the triangle inequality, which is another key property exploited in Euclidean shortest-path finding problems. In particular, it is not guaranteed that one of the 'taut-string' paths will be an optimal obstacle-avoiding path. Thus, the traditional approach of searching among a finite number of taut-string paths may fail to deliver an optimal solution.

It is important to note that the analysis and results presented in this paper apply to a wide range of optimal path finding problems, and not only to the problems of minimum travel time. Despite the fact that our discussion here is limited for ease of exposition to the direction-dependent speed functions, it can be easily extended to other anisotropic cost functions, such as fuel and energy consumption or agent's motions.

Finally, while the work presented in this paper makes a restrictive assumption of time and space homogenous environment, we relax this assumption in our forthcoming work and generalize the problem. This paper and its results are the fundamental building blocks of the more general models, and the analytical nature of this special case analysis is critical to fast and computationally efficient algorithms developed for time- and space-dependent problems.

\section{$1.1 \quad$ Related Work}

The majority of the optimal path planning work to date concentrates on determining Euclidean shortest paths (see survey [2]). A number of extensions have been considered (e.g., [3, 4]), yet most work is restricted to isotropic metrics. Some shortest path finding problems discussed in the literature [5,6] introduce direction dependency by restricting the feasible paths to a fixed set of orientations; however the resulting cost function retains its metric properties. Optimal path finding problems in anisotropic media have been addressed for a few specific applications, and the solution approach and results are often customized to the application at hand. For example, [7, 8] study optimal path finding for a mobile agent (e.g., robot or vehicle) across hilly terrains, where a physical model of friction and gravity forces is used to compute the anisotropic cost function for the agent.

In the area of optimal yacht sailing, Philpott et al. [9] create a mathematical programming model that evaluates the vessel speed for a specified range of wind speeds and yacht heading angles. The resulting data is used to find the yacht fastest path by applying dynamic programming algorithms [10, 11, 12]. Alternatively, Sellen [13] studies the optimal sailing routing problems for a more abstract scenario, and presents some results similar to ours by heuristically arguing that an optimal path in an obstacle-free domain consists of at most two line segments. Sellen's analysis is limited to problems with very specific speed functions represented by piecewise-linear reciprocal functions.

Some researchers have employed the calculus of variations and optimal control theory for optimal vessel routing problems. References $[14,15,16]$ employ Euler's equations to characterize an optimal path; while [17] establishes an analogy between a traveling light ray and an optimal path seeking sailboat, and extends the use of optical principles to sailing strategies. These optimal-path finding methods reduce to solving systems of differential equations, which can present a challenging task. Moreover, researchers typically use a simplified form of the speed function in order to make the analysis more manageable. From our experience of working on vessel routing problems [1], it is clear that analytical functions cannot always accurately describe vessel movement through waves, thus obliging us to look for alternative methods to solve the problem.

Reif and Sun [18] investigate a problem of time-optimum movement planning through a set of polygonal regions, where anisotropy is introduced as a uniform flow assigned to each region. The 
actual velocity of an object is defined to be the sum of a flow vector and a chosen control velocity. While the resulting speed function does display the direction-dependent property, its structure is very specific, and Reif and Sun's analysis does not extend to more general problems addressed in this paper.

In the most recent work on anisotropic movement, Cheng et al. [19] generalize the problem studied by Reif and Sun, and look at shortest path finding in anisotropic regions where the directiondependency of the speed is not restricted to the effect of the uniform flow. Cheng et al. still limit their research to the speed function with a very specific structure, referred to as a 'convex distance function' (first discussed in [20]). Their convex distance function is equivalent to our case of a convex linear path attainable region. The results presented in our work subsequently relax the convexity assumption and deliver a closed form fastest path among obstacles for a general anisotropic speed function. In addition, we provide rigorous proofs previously absent in the published work on convex distance functions.

\subsection{Overview of the Results}

This paper presents solution to the fastest-path finding problem for any given anisotropic speed function. We demonstrate that an optimal path in a general obstacle-free, time and space homogeneous medium is piecewise-linear with at most two line segments (i.e., one waypoint). Consequently, we merge these results with the visibility graph search methods developed for Euclidean shortest path problems [21, 22], to develop an obstacle-avoiding fastest-path finding algorithm for anisotropic speed function. Our results provide computationally fast techniques for finding a closed form solution to the very large class of applied problems discussed earlier.

While our main results make no assumptions about the structure of the speed function, we first consider a special case of the problem where the speed polar plot (or the linear path attainable region) encloses a convex region. This restricted scenario provides important insight and intuition to the structure of an optimal path for the more general case. Subsequently, we relax the convexity assumption to consider a case for a very general speed function. One of our main results is presented in Theorem 3.4, which characterizes a fastest path for an arbitrary speed function in an obstacle-free domain. Algorithm 3.1 describes a step-by-step procedure to construct such a path. In addition to characterizing a fastest path, we also compute a bound on the improvement in travel time were one to choose to follow an optimal path as opposed to traversing the simpler linear path between the two points. This bound is an important tool for evaluating tradeoffs, as well as for proving our key theorem.

We employ our findings for fastest path in an obstacle-free domain to the problems that consider the presence of polygonal obstacles. For the speed functions corresponding to convex linear path attainable regions, the straight line path is a fastest path in $\Re^{2}$, and the triangle inequality holds true in an obstacle-free domain. Consequently, fastest-path finding in a polygonal domain can be restricted to a modified visibility graph, similarly to Euclidian shortest-path finding problems. The triangle inequality might not hold true for a general speed function. In that case, an augmented speed function corresponding to the convex hull of the original speed polar plot is used to find a lower bound on the minimum travel time for our problem. We use the results for an optimal path in the obstacle-free domain to construct an obstacle-avoiding path that achieves this lower bound, thus establishing its optimality.

The rest of the paper is organized as follows. Section 2 provides the notation and a rigorous statement of the problem. Section 3 develops and presents fastest paths for anisotropic speed function in an obstacle-free domain. It also includes the description of Algorithm 3.1 that facilitates the implementation of the presented results. The following Section 4 extends our analysis and 
results to the obstacle-avoiding fastest-path finding problems in anisotropic domain. Algorithms 4.1 and 4.2 describe the fastest-path finding procedures corresponding to the case of a convex and generalized linear path attainable regions, respectively. Section 5 concludes this paper with an example of application of our results to the vessel routing problem, summarizes the findings and contribution of the presented work, and describes the future directions to extend this work. For conciseness and improved flow of the paper, some proofs are omitted from the text, and reader is referred to technical report [23] for more detailed discussion.

\section{Notation and Problem Statement}

Let $\mathcal{P} \subset \Re$ denote a set of open polygonal obstacles such that their closures do not intersect, and $\mathcal{F}:=\Re^{2} \backslash \mathcal{P}$ denote the free space where all the feasible paths mush lie. In the obstacle-free case we set $\mathcal{P}=\emptyset$. We define a set of feasible paths from $s$ to $t$ as $P_{s t}=\{p:[0,1] \rightarrow \mathcal{F}$ such that $p(0)=s, p(1)=t, p$ is continuous and rectifiable $\}$. Then, for any $p \in P_{s t}$, let $t(p)$ denote the travel time for path $p$.

Let $V(\theta)$ for $\theta \in[0,2 \pi]$ denote the maximum attainable speed for a given heading $\theta$. Unless otherwise specified, we assume that $V(\theta)>0, \forall \theta \in[0,2 \pi]$. The case where $V(\theta)=0$ for some $\theta$ is discussed separately in Section 3.3. Note that, without loss of optimality, one always travels at the maximum attainable speed.

We define $L_{\delta}(x)$ to be the linear path attainable region (LPAR) for a given point $x \in \Re^{2}$ and time $\delta>0$. That is, $L_{\delta}(x)=\left\{y \in \Re^{2}:\|y-x\| \leq \delta V\left(\theta_{y-x}\right)\right\}$, where $\theta_{y-x}$ and $\|y-x\|$ denote the angle and length of a vector $y-x$, respectively. Note that $L_{1}(0,0)$ is equivalent to a region enclosed by a polar graph of the speed function $V(\theta)$, and is often referred to as the 'speed polar plot'. Let $A_{\delta}(x)$ be the attainable region (AR), $A_{\delta}(x)=\left\{y: \exists p \in P_{x y}\right.$ such that $\left.t(p) \leq \delta\right\}$. In the presence of obstacles (i.e., $\mathcal{P} \neq \emptyset$ ) it is assumed that $x \notin \mathcal{P}$, and $\delta$ is too small to reach any obstacles from $x$.

Finally, we let the function $\tau(x, y): \Re^{2} \times \Re^{2} \rightarrow \Re^{+}$denote the travel time from point $x$ to point $y$ following the straight line path connecting these two points. We assume that $\{\lambda x+(1-\lambda) y$ : $\lambda \in[0,1]\} \cap \mathcal{P}=\emptyset$. Then $\tau(x, y)=\min \left\{\delta: y \in L_{\delta}(x), \delta>0\right\}$ or $\tau(x, y)=\|y-x\| / V\left(\theta_{y-x}\right)$. If $V\left(\theta_{y-x}\right)=0$, we set $\tau(x, y)=\infty$.

Problem statement: For a given speed function $V(\theta):[0,2 \pi] \rightarrow \Re^{+}$, a starting point $s \in \mathcal{F}$, and a target point $t \in \mathcal{F}$, find a fastest path from $s$ to $t$ that lies in $\mathcal{F}$. That is, our objective is to find $p^{*} \in P_{s t}$ such that $t\left(p^{*}\right) \leq t(p)$ for all $p \in P_{s t}$.

\section{Fastest-Path Finding in an Obstacle-Free Domain}

In this section we study the fastest-path finding problems in an obstacle-free domain, that is, $\mathcal{P}=\emptyset$ and $\mathcal{F}=\Re^{2}$.

\subsection{Fastest Path for a Convex Linear Path Attainable Region}

We first analyze a problem restricted to the convex LPAR and assume that $L_{\delta}(x)$ is convex for all $x$ and $\delta$. Let $m(x):=\inf \left\{r: \frac{x}{r} \in L_{1}(0,0), r>0\right\}$. Observe that since $L_{1}(0,0)$ is a closed set, the infimum is achieved and the definition can we rewritten as $m(x):=\min \left\{r: \frac{x}{r} \in L_{1}(0,0), r>0\right\}$ as long as $x \neq(0,0)$. Also, note that since $L_{1}(0,0)=L_{m(x)}(0,0) / m(x)$ is a convex set in $\Re^{2}$ and $(0,0)$ is its interior point, $m(x)$ is the Minkowski functional. We then know from [24] that the Minkowski functional $m\left(\right.$.) satisfies the inequality $m\left(x_{1}+x_{2}\right) \leq m\left(x_{1}\right)+m\left(x_{2}\right)$ for all $x_{1}, x_{2} \in \Re^{2}$. A couple of algebraic manipulations lead to the fact that $m(x)$ reduces to the straight line travel 


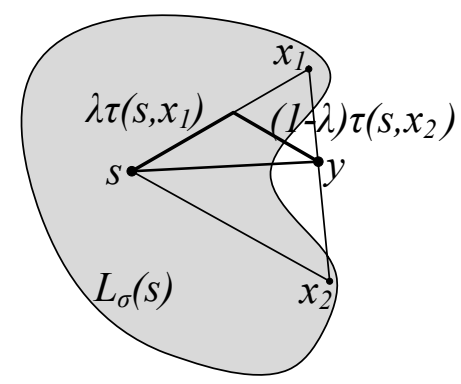

Figure 1: Theorem 3.1 counter example for a non-convex linear path attainable region.

time function $\tau($.$) , that is, \tau(x, y)=m(y-x)$ for all $x, y \in \Re^{2}$. Then we can show that the equivalent inequality holds true for the travel time function $\tau$. That is, for any $x, y, z \in \Re^{2}$, we have $\tau(x, y) \leq \tau(x, z)+\tau(z, y)$. We can use this triangle inequality to show that the straight line path between two points is a fastest path for a convex LPAR, $L_{\delta}(x)$. Furthermore, the convexity of an LPAR is also a necessary condition for the straight line path to be optimal, both of which facts are established in Theorem 3.1 (see Figure 1). (Reader is referred to [23] for this and following omitted proofs.)

Theorem 3.1. A fastest path in $\Re^{2}$ from an arbitrary start point $s \in \Re^{2}$ to any other point in $\Re^{2}$ is a path along the straight line connecting the two points if and only if the linear path attainable region $L_{\delta}(x)$ is a convex set for all $x \in \Re^{2}$.

It is important to acknowledge that earlier work on 'convex distance functions' $[25,20,19]$ have stated some results similar to Theorem 3.1. However, none of the found literature provides a rigorous proof in its entirety.

\subsection{Fastest Path for an Arbitrary Linear Path Attainable Region}

Next, we relax the convexity assumption for the linear path attainable region, $L_{\delta}(x)$, and analyze the problem for a general time and space homogeneous speed function. First, we analyze the properties of LPAR, $L_{\delta}(x)$, and the corresponding attainable region, $A_{\delta}(x)$. Consider any two points $x_{1}, x_{2} \in L_{\delta}(x)$. Then, for any point $y=\lambda x_{1}+(1-\lambda) x_{2}$, for $\lambda \in[0,1]$, we can construct a piecewise linear path $p$ consisting of segments $\left(x, \lambda x_{1}\right)$ and $\left(x,(1-\lambda) x_{2}\right)$ (see Figure 1$)$. Since the travel time for such path $p$ is less than or equal to $\delta$, we can conclude that $y \in A_{\delta}(x)$. Thus, the convex combination of any two points in $L_{\delta}(x)$ is contained in $A_{\delta}(x)$, i.e., $\forall x_{1}, x_{2} \in L_{\delta}(x)$ and $\forall \lambda \in[0,1], \lambda x_{1}+(1-\lambda) x_{2} \in A_{\delta}(x)$. This leads to Theorem 3.2.

Theorem 3.2. Attainable region, $A_{\delta}(x)$, is the convex hull of the corresponding linear path attainable region, $L_{\delta}(x)$, i.e., $A_{\delta}(x)=\operatorname{conv}\left(L_{\delta}(x)\right)$.

Now, we can compute a bound on the decrease in the travel time from point $x$ to point $y$ by following an optimal path instead of the straight line path, without actually knowing the optimal path. Consider a non-convex linear path attainable region, $L_{\delta}^{1}(x)$, corresponding to some speed function $V^{1}(\theta)$, and a new linear path attainable region defined as the convex hull of the original LPAR, that is, $L_{\delta}^{2}(x)=\operatorname{conv}\left(L_{\delta}^{1}(x)\right)$. Let $V^{2}(\theta)$ be the maximum attainable speed function associated with the new LPAR. From Theorem 3.1, we know that for $L_{\delta}^{2}(x)$, the fastest path from $x$ to $y$ is along the straight line segment connecting these two points, $l_{x y}$, with the total travel time $\tau_{2}(x, y)=\|y-x\| / V^{2}\left(\theta_{y-x}\right)$. Since $L_{\delta}^{1}(x) \subset L_{\delta}^{2}(x)$, we know that $V^{1}(\theta) \leq V^{2}(\theta), \forall \theta$. Then, the 


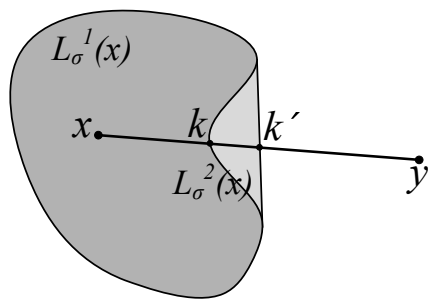

Figure 2: Computing a bound on the decrease in travel time for a non-convex linear path attainable region, $L_{\delta}^{1}(x)$.

smallest travel time from $x$ to $y$ for the linear path attainable region $L_{\delta}^{1}(x)$, denoted by $t_{L_{\delta}^{1}(x)}^{*}(x, y)$, is at least as much $\tau_{2}(x, y)$, i.e., $t_{L_{\delta}^{1}(x)}^{*}(x, y) \geq \tau_{2}(x, y)$. Define $k$ to be the point of intersection of the line connecting points $x$ and $y$, and the boundary of the linear path attainable region $L_{\delta}^{1}(x)$, i.e., $k:=l_{x y} \cap b d\left(L_{\delta}^{1}(x)\right)$. Similarly, we define $k^{\prime}:=l_{x y} \cap b d\left(L_{\delta}^{2}(x)\right)$ (see Figure 2). Note that the sets $L_{\delta}^{1}(x)$ and $L_{\delta}^{2}(x)$ are closed and therefore contain their boundaries. Also note that the travel time along the straight line path from $x$ to $y$ corresponding to the linear path attainable region $L_{\delta}^{1}(x)$ is $\tau_{1}(x, y)=\delta \frac{\|y-x\|}{\|k-x\|}$. Set $\beta:=\frac{\|k-x\|}{\left\|k^{\prime}-x\right\|} \leq 1$. Then, we have the following bounds on $t_{L_{\delta}^{1}(x)}^{*}(x, y)$.

$$
\begin{aligned}
\tau_{2}(x, y) \leq t_{L_{\delta}^{1}(x)}^{*}(x, y) & \leq \tau_{1}(x, y) \\
\delta \frac{\|y-x\|}{\left\|k^{\prime}-x\right\|} \leq t_{L_{\delta}^{1}(x)}^{*}(x, y) & \leq \delta \frac{\|y-x\|}{\|k-x\|} \\
\beta \delta \frac{\|y-x\|}{\|k-x\|} & \leq t_{L_{\delta}^{1}(x)}^{*}(x, y) \leq \delta \frac{\|y-x\|}{\|k-x\|} \\
\beta \tau_{1}(x, y) \leq t_{L_{\delta}^{1}(x)}^{*}(x, y) & \leq \tau_{1}(x, y)
\end{aligned}
$$

From inequalities (1), we deliver the following proposition.

Proposition 3.3. The optimal travel time for a non-convex LPAR is at most $\beta$ times shorter than following a straight line path from $x$ to $y$, where $\beta:=\frac{\|k-x\|}{\left\|k^{\prime}-x\right\|}$. That is, the traveling time would at most decrease by 100(1- $\beta$ ) percent, if one were to follow an optimal path instead of traveling along the straight line.

This lower bound leads to Theorem 3.4, that establishes analytical characterization on a optimal path of any direction depended speed function, by demonstrating that the proposed path is, in fact, optimal.

Theorem 3.4. Consider a linear path attainable region $L_{\delta}(x)$. For two arbitrarily given points $x, y \in \Re^{2}$, let $k$ denote the intersection point of the line connecting $x$ and $y, l_{x y}$, and the boundary of the set $L_{\delta}(x)$, i.e., $k:=l_{x y} \cap b d\left(L_{\delta}(x)\right)$. Similarly, let $k^{\prime}:=l_{x y} \cap b d\left(\operatorname{conv}\left(L_{\delta}(x)\right)\right)$. Then, the fastest path from $x$ to $y$ is described by one of the following two scenarios.

1. If $k=k^{\prime}$, the fastest path from $x$ to $y$ is the straight line segment connecting these two points (Figure 3, case $y=y_{1}$ ).

2. If $k \neq k^{\prime}$, the fastest path from $x$ to $y$ consists of two line segments: the straight line segment from point $x$ to point $z=x+\alpha \lambda^{*}\left(x_{1}-x\right)$ and the second line segment from point $z$ to point $y$, where $\alpha=\frac{\|y-x\|}{\left\|k^{\prime}-x\right\|}$ and $x_{1}, x_{2} \in L_{\delta}(x)$ s.t. $\exists \lambda^{*} \in[0,1]: k^{\prime}=\lambda^{*} x_{1}+\left(1-\lambda^{*}\right) x_{2}$. (See Figure 3 , case $y=y_{2}$, and note that $\left.\left(y_{2}-z\right) \|\left(x_{2}-x\right)\right)$. 


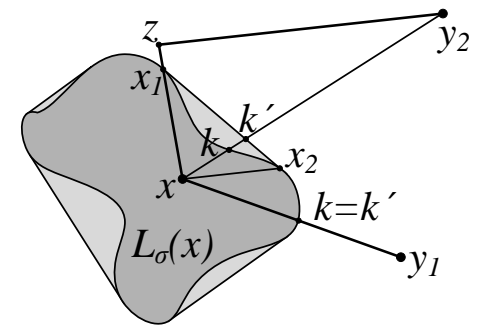

Figure 3: Illustration of Theorem 3.4: scenario $1\left(k=k^{\prime}\right.$ for $\left.y_{1}\right)$ and scenario $2\left(k \neq k^{\prime}\right.$ for $\left.y_{2}\right)$.

Proof. 1. Consider the case where $k=k^{\prime}$. From inequalities $(1)$, we have $\beta \tau(x, y) \leq t_{L_{\delta}(x)}^{*}(x, y) \leq$ $\tau(x, y)$, where $\beta:=\frac{\|k-x\|}{\left\|k^{\prime}-x\right\|}$ and $t_{L_{\delta}(x)}^{*}(x, y)$ is the minimum travel time from $x$ to $y$. Since $k=k^{\prime}$, we have $\beta=1$, and $\tau(x, y) \leq t_{L_{\delta}(x)}^{*}(x, y) \leq \tau(x, y) \Rightarrow t_{L_{\delta}(x)}^{*}(x, y)=\tau(x, y)$. This means that the travel time from $x$ to $y$ along the straight line path equals the minimum travel time, and hence, straight line path is a fastest path from $x$ to $y$.

2. Now, we consider the case where $k \neq k^{\prime}$. From definition of $k^{\prime}$, we have that $k^{\prime} \in \operatorname{conv}\left(L_{\delta}(x)\right)$. Then, $\exists \lambda^{*} \in[0,1]$ and $\exists x_{1}, x_{2} \in L_{\delta}(x)$, such that $\lambda^{*} x_{1}+\left(1-\lambda^{*}\right) x_{2}=k^{\prime}$. Note, that since $x_{1}, x_{2} \in L_{\delta}(x)$, we know that $\tau\left(x, x_{1}\right) \leq \delta$ and $\tau\left(x, x_{2}\right) \leq \delta$.

From inequalities (1), we have $t_{L_{\delta}(x)}^{*}(x, y) \geq \delta \frac{\|y-x\|}{\left\|k^{\prime}-x\right\|}=\delta \alpha$, where $t_{L_{\delta}(x)}^{*}(x, y)$ is the minimum travel time from $x$ to $y$. Now, consider the following path $p$ : from point $x$ we follow vector $\alpha \lambda^{*}\left(x_{1}-x\right)$, and then, continue on following vector $\alpha\left(1-\lambda^{*}\right)\left(x_{2}-x\right)$. Note, that the first part of the path is equivalent to following a straight line segment from point $x$ to point $x+\alpha \lambda^{*}\left(x_{1}-x\right)=z$. And the second part of the path ends at point $x+\alpha \lambda^{*}\left(x_{1}-x\right)+\alpha(1-$ $\left.\lambda^{*}\right)\left(x_{2}-x\right)=x+\alpha\left(\left(\lambda^{*}\right)\left(x_{1}-x\right)+\left(1-\lambda^{*}\right)\left(x_{2}-x\right)\right)=x+\alpha\left(k^{\prime}-x\right)=y$. Hence, the proposed path $p$ is the same path as in the statement of the theorem. This proves the existence of the path described in the theorem.

Next, we want to find the travel time along this path $p, t(p)$. From the space and time homogeneity property, we have $t(p)=\alpha \lambda^{*} \tau\left(x, x_{1}\right)+\alpha\left(1-\lambda^{*}\right) \tau\left(x, x_{2}\right) \leq \alpha \lambda^{*} \cdot \delta+\alpha\left(1-\lambda^{*}\right) \cdot \delta=$ $\alpha \delta$. Since travel time for path $p$ is less than or equal to the lower bound on the minimum travel time from $x$ to $y$ (i.e., $\left.t(p) \leq t_{L_{\delta}(x)}^{*}(x, y)\right), t(p)$ must be equal to the minimum travel time from $x$ to $y$. Hence, our path $p$ is, in fact, a fastest path from $x$ to $y$.

It is worth noting that in the case when $k \neq k^{\prime}$ (corresponding to scenario 2 of Theorem 3.4) the fastest path constructed in the theorem is not uniquely optimal. It is only one of the infinitely many feasible paths with the same minimum travel time. Note that any zigzag path from $x$ to $y$ restricted to the traveling directions of the vectors $x_{1}-x$ and $x_{2}-x$ would correspond to the same minimum travel time. Furthermore, the straight line path in the case of $k=k^{\prime}$ might also not be uniquely optimal. Depending on the structure of the speed function, it is possible that a piecewise-linear path would have the same optimal travel time as the straight line. 


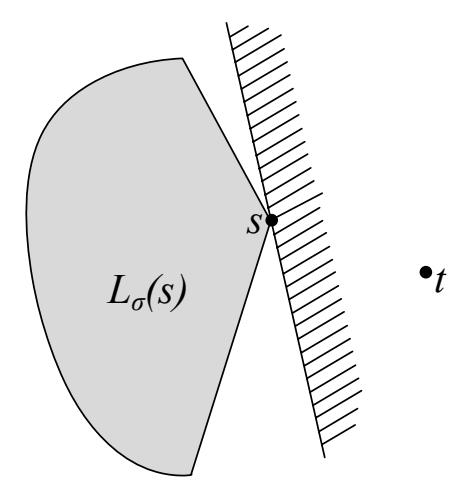

Figure 4: Example of a convex LPAR where $V\left(\theta_{s t}\right)=0$; there is no feasible path from $s$ to $t$.

\subsection{Problem Feasibility and Fastest-Path Finding for a Non-negative Speed Function}

Thus far we assumed that $V(\theta)>0$ for all $\theta \in[0,2 \pi]$. However in practice, the speed function $V(\theta)$ can take on the value of zero for some headings, i.e., leading to a 'stall'. For example, a vehicle traveling across some hilly terrain might encounter impermissible headings due to overturn danger or power limitations [7]. On another hand, a sailing boat can not travel in head sea corresponding to a zero speed for that heading [11]. In this section, we discuss the case where $V(\theta) \geq 0, \forall \theta \in[0,2 \pi]$. To avoid the trivial case, we assume $V(\theta)>0$ for some $\theta$.

Similarly to a positive speed function case, we first analyze the case where $\operatorname{LPAR}, L_{\delta}(s)$, is convex. Observe that if $V(\theta)=0$ for some $\theta \in[0,2 \pi]$, and the corresponding linear path attainable region, $L_{\delta}(s)$, is convex, then there exists a line passing through the starting point $s$ such that none of the points belonging to one of the half-planes created by this line can be reached (see Figure 4). Combining this with Theorem 3.1 we establish Theorem 3.5 (Recall that $\theta_{s t}$ denotes the heading angle of the vector $t-s$. ).

Theorem 3.5. Assume that LPAR, $L_{\delta}(s)$, corresponding to some speed function $V(\theta) \geq 0$, is convex. Then,

1. if $V\left(\theta_{\text {st }}\right)=0$, a feasible path from $s$ to $t$ does not exist; and

2. if $V\left(\theta_{s t}\right)>0$, a fastest path from $s$ to $t$ is along the straight line path st.

We now relax the convexity assumption and analyze optimal paths for a general $L_{\delta}(s)$. In the case when $V\left(\theta_{s t}\right)>0$, the optimal path finding problem is always feasible, and when $V\left(\theta_{s t}\right)=0$, the feasibility condition can be established as follows. Assume $V\left(\theta_{s t}\right)=0$, and define $\underline{\theta}$ and $\bar{\theta}$ as

$$
\begin{aligned}
& \underline{\theta}=\inf \left\{\theta^{*}: V(\theta)=0, \forall \theta \in\left[\theta^{*}, \theta_{s t}\right]\right\} \\
& \bar{\theta}=\sup \left\{\theta^{*}: V(\theta)=0, \forall \theta \in\left[\theta_{s t}, \theta^{*}\right]\right\} .
\end{aligned}
$$

In defining $\underline{\theta}$ and $\bar{\theta}$ we extend the domain of the speed function to $[-\pi, 3 \pi]$, by observing that $V(\theta)=V(\theta+2 \pi), \forall \theta$, to guaranty the continuity of the interval. Then we can show (see [23]) that a feasible path from $s$ to $t$ does not exist if and only if $V\left(\theta_{s t}\right)=0$ and $\bar{\theta}-\underline{\theta} \geq \pi$. Finally, Theorem 3.6 delivers an optimal path from $s$ to $t$ for a general linear path attainable region.

Theorem 3.6. Consider $V(\theta) \geq 0$ for all $\theta \in[0,2 \pi]$, and let $L_{\delta}(s)$ be the corresponding linear path attainable region. Then, 


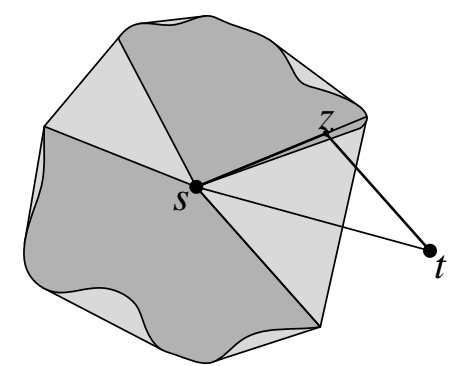

Figure 5: An optimal path from $s$ to $t, s z t$.

1. if $V\left(\theta_{s t}\right)=0$ and $\bar{\theta}-\underline{\theta} \geq \pi$, a feasible path from $s$ to $t$ does not exist; and

2. if $V\left(\theta_{s t}\right)>0$ or $\bar{\theta}-\underline{\theta}<\pi$, then a fastest path from $s$ to $t$ is characterized the same way as in Theorem 3.4, where $x=s, y=t$ and if $V\left(\theta_{\text {st }}\right)=0$ we set $k=s$. (See Figure 5.)

\subsection{Fastest-Path Finding Algorithm}

In this section, we discuss the implementation of the results presented in previous sections and provide an algorithm that can be implemented by a computer program (e.g., on-board autonomous navigation system) to find a fastest path from a given start point $s \in \Re^{2}$ to a given target point $t \in$ $\Re^{2}$. We assume that the values of a speed function $V(\theta)$ are given for a discrete set of equally spaced heading angles, $\theta$, which we denote by the set of polar coordinates $S=\left\{\left(\theta_{0}, V\left(\theta_{0}\right), \ldots,\left(\theta_{n}, V\left(\theta_{n}\right)\right\}\right.\right.$, (see Figure 6 for an example).

The first step in finding a fastest path is to construct a convex hull of the linear path attainable region. We recommend the use of Graham's Scan algorithm [26, 27] to accomplish this task, since it uses a "rotational sweep" technique, processing vertices in the order of polar angles they form with a reference vertex. The polar nature of our LPAR makes Graham's Scan a favorable choice as it forgoes the sorting procedure required for other algorithms.

After the construction of a convex hull, we obtain a subset $S^{\prime} \subseteq S$ corresponding to the extreme points of the resulting convex hull. Let $l_{s t}$ denote the straight line passing through points $s$ and $t$, and $\theta_{s t}$ the heading angle of the vector $t-s$. Then, we need to find the point of intersection of $l_{s t}$ with the boundary of $L_{1}(s)$, denoted by $k$, and the point of intersection of $l_{s t}$ with the boundary of a convex hull of $L_{1}(s)$, denoted by $k^{\prime}$. To do so, we find between which two headings in sets $S$ and $S^{\prime}$ our $\theta_{s t}$ falls. We label such headings as $\theta_{L}$ and $\theta_{U}$, and $\theta_{L}^{\prime}$ and $\theta_{U}^{\prime}$, respectively (See Figure $6, L$ and $U$ stand for the lower and upper headings). After some algebraic manipulations omitted here, we compute the values of $\alpha$ and $\lambda^{*}$, as defined in Theorem 3.4 scenario 2 , to be

$$
\alpha \lambda^{*}=\frac{\|t-s\| \sin \left(\theta_{U}^{\prime}-\theta_{s t}\right)}{V\left(\theta_{L}^{\prime}\right) \sin \left(\theta_{U}^{\prime}-\theta_{L}^{\prime}\right)} .
$$

Then, we know that a fastest path is piecewise-linear with a $\operatorname{single~waypoint~} z=s+\alpha \lambda^{*}\left(\cos \left(\theta_{U}^{\prime}\right), \sin \left(\theta_{U}^{\prime}\right)\right)$.

The following algorithm outlines a step-by-step procedure of finding the fastest path from $s$ to $t$.

Algorithm 3.1. Fastest Path from $s$ to $t$ in an Obstacle-Free Domain.

Step 1. Find $\underline{\theta}$ and $\bar{\theta}$ using equations (2) and (3).

If $V\left(\theta_{s t}\right)=0$ and $\bar{\theta}-\underline{\theta} \geq \pi, S T O P$. The problem is infeasible.

Else, go to step 2. 


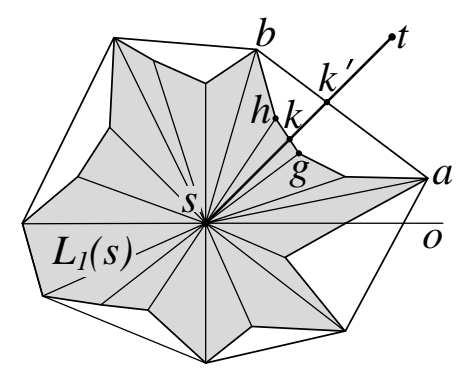

Figure 6: $L_{1}(s)$ and its convex hull: $\angle o s t=\theta_{s t}, \angle o s g=\theta_{L}, \angle o s h=\theta_{U}, \angle o s a=\theta_{L}^{\prime}$, and $\angle o s b=\theta_{U}^{\prime}$.

Step 2. Find the convex hull of the linear path attainable region $L_{1}(s)$.

Step 3. Find the heading angle $\theta_{\text {st }}$ and compute the values of $\theta_{L}, \theta_{U}, \theta_{L}^{\prime}$ and $\theta_{U}^{\prime}$.

Step 4. If points $\left(\theta_{L}, V\left(\theta_{L}\right)\right),\left(\theta_{U}, V\left(\theta_{U}\right)\right),\left(\theta_{L}^{\prime}, V\left(\theta_{L}^{\prime}\right)\right)$ and $\left(\theta_{U}^{\prime}, V\left(\theta_{U}^{\prime}\right)\right)$ are collinear (i.e., $\left.k=k^{\prime}\right)$, STOP. Straight line path st is an optimal path.

Else (i.e., $k \neq k^{\prime}$ ), go to step 5.

Step 5. Compute $\alpha \lambda^{*}$ using equation (4).

Set $z=s+\alpha \lambda^{*}\left(\cos \left(\theta_{U}^{\prime}\right), \sin \left(\theta_{U}^{\prime}\right)\right) \in \Re^{2}$. A fastest path from $s$ to $t$ is the two consecutive straight line segments $s z$ and then $z t$.

\section{Obstacle-Avoiding Fastest-Path Finding}

In this section we discuss obstacle-avoiding fastest-path finding by relaxing the assumption $\mathcal{P}=\emptyset$.

\subsection{Fastest Path for a Convex Linear Path Attainable Region}

Similarly to the analysis of path finding problems in an obstacle-free domain, we first restrict our attention to problems with convex LPARs. The visibility graph search method [2, 21, 22], used to solve Euclidean shortest-path finding problems with polygonal obstacles, exploits the triangle inequality property of the distance function and restricts the optimal path search to the set of 'taut strings' connecting the points of origin and destination. In the case of minimizing Euclidean distance, "an easy geometric argument shows that in general the shortest path between two points must be a polygonal chain whose vertices are vertices of obstacles" [22]. A similar observation is true for our anisotropic medium, and we can show (see [23]) that if a linear path attainable region $L_{\delta}(x)$ is convex, then there exists a fastest path from $s$ to $t$ in $\mathcal{F}$, which is piecewise-linear with all its waypoints (vertices) corresponding to the vertices of obstacles in $\mathcal{P}$. This implies that a fastest-path search can be restricted to a directed visibility graph with the edge cost defined to be the travel time along the straight line connecting its nodes. Henceforth, we adapt the shortest path visibility graph approach to develop the algorithm below.

Algorithm 4.1. Obstacle-Avoiding Fastest Path for a Speed Function V $(\theta)$ Corresponding to a Convex LPAR.

Step 1. Construct a visibility graph $\mathcal{V G}_{V}$ as follows.

- The set of $\mathcal{V G}_{V}$ vertices is composed of all the vertices of the obstacles in $\mathcal{P}$, as well as points $s$ and $t$. 


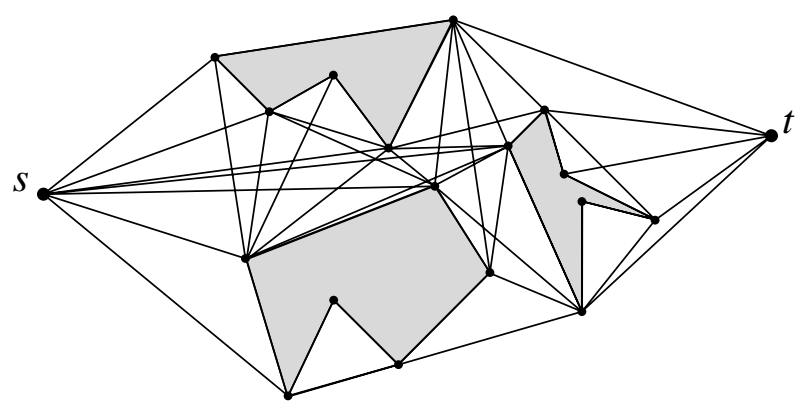

Figure 7: Example of constructing a visibility graph illustrating all the visibility graph nodes and edges

- The set of $\mathcal{V G}_{V}$ edges consists of all the straight line edges interconnecting these vertices such that they do not intersect any of the obstacles in $\mathcal{P}$ (see Figure 7).

- The cost associated with an edge $(i, j)$ is equal to the travel time $\tau(i, j)=\|j-i\| / V\left(\theta_{j-i}\right)$.

Step 2. Find a minimum cost path in the constructed network $\mathcal{V G}_{V}$ from node $s$ to node $t$. The resulting path is an obstacle-avoiding fastest path in $\mathcal{F}$.

Note that unlike the case of Euclidean metric, our visibility graph has to be directed since the cost of an $\operatorname{arc}(i, j)$ does not generally equal to the cost of an $\operatorname{arc}(j, i)$.

\subsection{Fastest Path for an Arbitrary Anisotropic Speed Function}

Subsection 4.1 discusses a direct extension of the shortest-path visibility graph approach to the obstacle-avoiding fastest-path finding problems with convex LPAR. In general, the triangle inequality does not hold true for the travel time function $\tau($.$) , and we cannot restrict our fastest-path$ search to the set of taut strings connecting $s$ and $t$. Here, we relax the convexity assumption of an LPAR, and analyze fastest-path finding problems for a general anisotropic speed function.

Consider an arbitrary speed function $V(\theta)$ and the corresponding linear path attainable region $L_{\delta}(x)$. We introduce an augmented speed function $V^{\prime}(\theta)$, such that, its corresponding LPAR, $L_{\delta}^{\prime}(x)$, is the convex hull of $L_{\delta}(x)$, i.e., $L_{\delta}^{\prime}(x):=\operatorname{conv}\left(L_{\delta}(x)\right)$. By definition, $L_{\delta}^{\prime}(x)$ is convex. Therefore, by constructing the visibility graph $\mathcal{V G}_{V^{\prime}}$ as described in Algorithm 4.1, we can find an obstacle-avoiding fastest path from $s$ to $t$ corresponding to the new speed function $V^{\prime}(\theta)$. We let $p_{V^{\prime}}$ represent this optimal path and $t_{V^{\prime}}\left(p_{V^{\prime}}\right)$ denote the travel time along the path $p_{V^{\prime}}$ while traveling with speed $V^{\prime}(\theta)$. Then, the travel time along an optimal path, $\left(p_{V}\right)$, from $s$ to $t$ corresponding to the original speed function $V(\theta)$ has to be greater than or equal to $t_{V^{\prime}}\left(p_{V^{\prime}}\right)$, i.e., $t_{V}\left(p_{V}\right) \geq t_{V^{\prime}}\left(p_{V^{\prime}}\right)$ [23]. This provides a lower bound on the minimum travel time for our original problem, which we use to demonstrate that a proposed path is optimal.

Since path $p_{V^{\prime}}$ lies in the visibility graph $\mathcal{V G}_{V^{\prime}}$, it is piecewise-linear with the waypoints corresponding to the vertices of $\mathcal{P}$, and points $s$ and $t$. Consequently, the total travel time of the path can be written as the sum of travel times along each individual link. Recall that the travel time for each linear link $(i, j)$ of the path $p_{V^{\prime}}$ is equal to $\tau^{\prime}(i, j)=\frac{\|j-i\|}{V^{\prime}\left(\theta_{j-i}\right)}$. From Theorem 3.4, our obstacle-free analysis describes a fastest path from $i$ to $j$ for an arbitrary speed function $V(\theta)$ with the optimal travel time equal to $\tau^{\prime}(i, j)$. Applying the theorem to each linear link of the path $p_{V^{\prime}}$ and then combining them together results in a path corresponding to the original speed function $V(\theta)$ with the travel time equal to the lower bound $t_{V^{\prime}}\left(p_{V^{\prime}}\right)$. Recall that an optimal path 


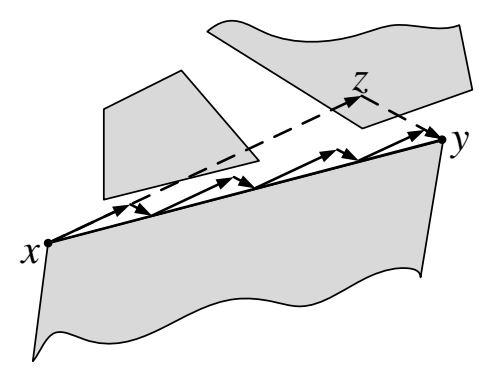

Figure 8: A feasible zigzag path from $x$ to $y$ with the total travel time equal to $\tau^{\prime}(x, y)$.

described in the second scenario of Theorem 3.4 is not unique, and we can construct a zigzag path with minimum travel time by alternating the traveling directions between headings corresponding to vectors $x_{1}-x$ and $x_{2}-x$. Since we assume that the distance between any two obstacles is greater than zero, we can construct a zigzag path close enough to the line $x y$, such that it does not intersect with the neighboring obstacles. (See Figure 8.)

Algorithm 4.2. Obstacle-Avoiding Fastest Path for an Arbitrary Speed Function $V(\theta)$.

Step 1. Find $V^{\prime}(\theta)$ for $\theta \in[0,2 \pi]$ such that $L_{\delta}^{\prime}(x)=\operatorname{conv}\left(L_{\delta}(x)\right)$.

Step 2. Use Algorithm 4.1 to find an optimal path corresponding to the speed function $V^{\prime}(\theta)$. Let $p_{V^{\prime}}$ denote the determined path, and let $\left(k_{0}, k_{1}, k_{2}, \ldots, k_{n}\right)$ be the sequence of vertices path $p_{V^{\prime}}$ is traversing. Note that $k_{0}=s$ and $k_{n}=t$. Then the corresponding travel time along the path $p_{V^{\prime}}$, denoted by $t_{V^{\prime}}\left(p_{V^{\prime}}\right)$, can we written as

$$
t_{V^{\prime}}\left(p_{V^{\prime}}\right)=\Sigma_{i=1}^{n} \tau^{\prime}\left(k_{i-1}, k_{i}\right)
$$

Step 3. For each pair of consecutive points in $\left(k_{0}, k_{1}, \ldots, k_{n}\right)$, apply Algorithm 3.1 to find a fastest path between the two points corresponding to the speed function $V(\theta)$. If a given one waypoint path is infeasible due to the presence of obstacles, increase the number of waypoints in a zigzag path as discussed above.

Step 4. Combine together the optimal paths found in Step 3. The resulting path has a travel time equal to $t_{V^{\prime}}\left(p_{V^{\prime}}\right)$ and is therefore a fastest obstacle-avoiding path for an arbitrary speed function $V(\theta)$. (See Figure 9.)

\section{Applications and Conclusion}

\subsection{Application: Optimal Short-Range Routing of Vessels in a Seaway}

A fastest-path finding problem for the direction-dependent speed functions arises in a wide range of applications. For example, the speed of a sail boat depends on the traveling heading angle it makes with wind, and a vehicle speed varies as the agent traverses up and down a hill. Airplanes have to deal with an anisotropic speed due to wind, while motor boats have similar effects caused by waves. To demonstrate an application in more details, we analyze optimal short-range routing of vessels in a seaway.

Any vessel traveling at a seaway encounters waves which add drag and affect the vessel's performance. In our collaboration with colleagues working on Optimal Vessel Performance in Evolving 


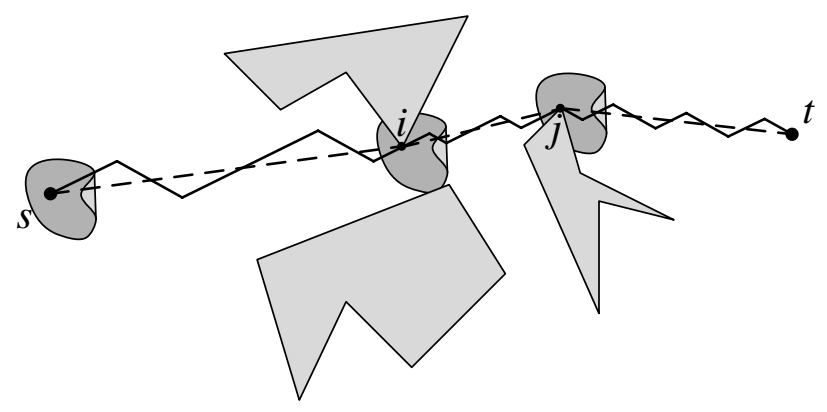

Figure 9: Example of a fastest path for speed function $V^{\prime}(\theta)$ (dashed line), and an optimal path for speed $V(\theta)$ (solid line).

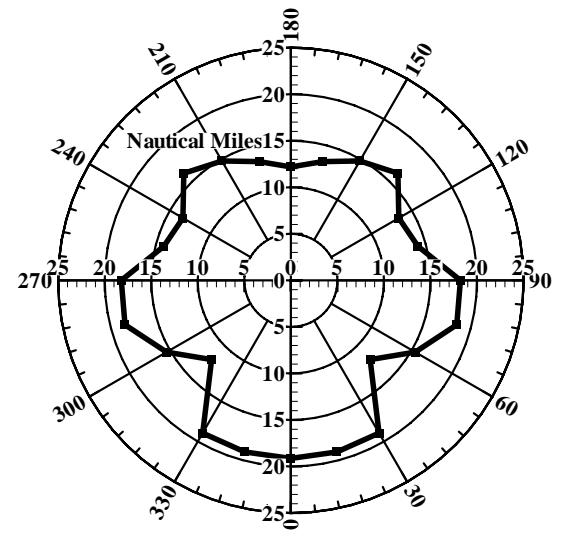

Figure 10: "An example of linear path attainable regions for the S-175 corresponding to voluntary speed loss at Sea State no.7" [1].

Nonlinear Wave-Fields project [1], we evaluate the added drag by computing the time average wave force acting on the vessel in the longitudinal direction. Then, by superimposing the added drag on the steady drag experienced by the moving ship in calm waters, we compute the maximum mean attainable speed for each given sea state (which describes the distribution of the waves) and the heading angles in the range from $0^{\circ}$ to $180^{\circ}$. Figure 10, borrowed from [1], illustrates an example of the linear path attainable region for the S-175 containership at Sea State no.7. Here, heading is measured as the angle a vessel makes with the dominant wave direction, which is assumed to be in the southerly direction.

For the given LPAR, we can use Theorem 3.4 to find a fastest path; Algorithm 3.1 describes the step-by-step procedure to construct such an optimal path. As an example, we consider two scenarios. In first case, let the target point $t_{1}$ lie directly east from the starting point $s$. This example corresponds to the scenario 1 of Theorem 3.4, since the straight line $s t_{1}$ intersects the boundary of the linear path attainable region $L_{\delta}(s)$ and the boundary of its convex hull at the same point. Hence, we can conclude that the straight line path $s t_{1}$ is a fastest path from $s$ to $t_{1}$, illustrated in Figure 11.

In the second example, let the target point $t_{2}$ lie south-west from the starting point $s$. Then, the intersection points of the line $s t_{2}$ with the boundary of $L_{\delta}(s)$ and the boundary of $L_{\delta}(s)$ 's convex hull are not the same (i.e., $k \neq k^{\prime}$ ), corresponding to the scenario 2 of Theorem 3.4; and 


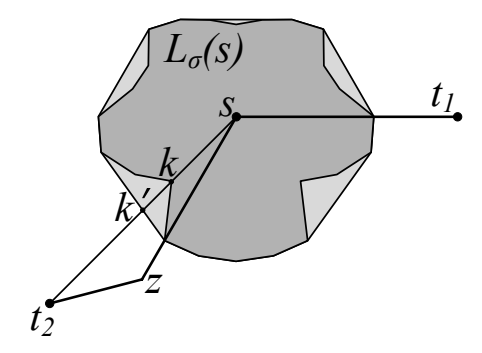

Figure 11: Illustration of the fastest paths from point $s$ to points $t_{1}$ and $t_{2}$, paths $s t_{1}$ and $s z t_{2}$, respectively.

a fastest path from $s$ to $t_{2}$ is piecewise-linear with one waypoint. Thus, to reach the point $t_{2}$ as fast as possible, the vessel should first travel SSE, or $30^{\circ}$ clockwise from the south direction, and then complete the travel heading $75^{\circ}$ clockwise from south. This corresponds to the path $s z t_{2}$, illustrated on the Figure 11.

In addition to finding a fastest path from $s$ to $t_{2}$, we can use equations (1) to calculate how much improvement in travel time a vessel observes as it follows the optimal path $s z t_{2}$ instead of following a straight line path $s t_{2}$. By dividing the length of $s k$ by the length of $s k^{\prime}$, we find that $\beta=0.688$, which implies that by following an optimal path we can decrease our travel time at most by approximately $31.2 \%$. This kind of information is particulary useful in evaluating the tradeoffs between following an optimal path as opposed to following a straight line.

In some applications seaway regions might be restricted for vessel's use due to severe weather, presence of land, other vessels, or imposed regulations. For such problems we approximate the restricted regions with polygonal obstacles and apply Algorithm 4.2 to find an optimal obstacleavoiding path to the destination. In other applications the vessel's speed is often maintained constant by utilizing a greater amount of fuel and varying the engine thrust level. Our solution approach easily extends to such problems. We redefine the linear path attainable region to represent the set of points one can reach consuming a single unit of fuel. Then, by using the algorithms presented in this paper we can find a path minimizing fuel consumption instead of traveling time.

\subsection{Conclusion and Discussion}

We find the solution to a fastest-path finding problem for a direction-dependent time and space homogeneous speed function. We demonstrate that in an obstacle-free domain an optimal path is piecewise-linear with at most two line segments, regardless of the underline structure of the speed function. This analytical character of our results provides a computationally fast method for finding an optimal path, making it suitable for online applications. We also provide a tight bound on the improvement in travel time by following an optimal path as opposed to traversing a simpler straight line path. Algorithm 3.1 presented in the paper facilitates a simple implementation of these results.

We also use these results to address the obstacle-avoiding fastest-path problems in anisotropic media. We use the properties of speed functions with the convex polar plots to adapt the visibility graph search method, traditionally used for Euclidean shortest-path problems, to find a solution for our type of problems. We then address the case of an arbitrary speed function, by introducing an augmented speed function such that its polar plot is the convex hull of the original speed plot. Then, we apply fastest piecewise-linear paths between the nodes of the visibility graph and construct a path with the travel time equal to its lower bound, thus establishing its optimality. We present the algorithms that give the detailed steps to finding an optimal obstacle-avoiding path 
for the convex speed polar plots (Algorithm 4.1) and a general time and space homogeneous speed function (Algorithm 4.2). We discuss the application of the results for optimal vessel routing in a seaway. The numerical example demonstrates over 30\% decrease in vessel travel time when our path-finding algorithm is implemented instead of a straight line path.

While the presented work makes a restrictive assumption of time and space homogeneity of the cost function, these results are critical components to our solution approach for more general problem settings. In our forthcoming work, we relax the assumption of time and space homogeneity and integrate the analytical results of this paper to deliver an efficient path-finding algorithm for the generalized problem. We also extend these results to the problem of finding a fastest curvatureconstrained path in an anisotropic environment, where instantaneous hading change (e.g., waypoint) is prohibited.

\section{Acknowledgment}

This paper is the result of work partially sponsored by the Office of Naval Research through the Multidisciplinary University Research Initiative (MURI) Optimal Vessel Performance in Evolving Nonlinear Wave Fields under contract N00014-05-1-0537.

\section{References}

[1] I. S. Dolinskaya, M. Kotinis, M. G. Parsons, and R. L. Smith. Optimal short-range routing of vessels in a seaway. Journal of Ship Research, 53(3):121-129, September 2009.

[2] Joseph S. B. Mitchell. Geometric shortest paths and network optimization. In Handbook of computational geometry, pages 633-701. North-Holland, Amsterdam, 2000.

[3] Joseph S. B. Mitchell and Christos H. Papadimitriou. The weighted region problem: finding shortest paths through a weighted planar subdivision. J. Assoc. Comput. Mach., 38(1):18-73, 1991.

[4] Zheng Sun and John H. Reif. On finding approximate optimal paths in weighted regions. $J$. Algorithms, 58(1):1-32, 2006.

[5] P. Widmayer, Y. F. Wu, and C. K. Wong. On some distance problems in fixed orientations. SIAM J. Comput., 16(4):728-746, 1987.

[6] Joseph S. B. Mitchell. $L_{1}$ shortest paths among polygonal obstacles in the plane. Algorithmica, 8(1):55-88, 1992.

[7] Neil C. Rowe and Ron S. Ross. Optimal grid-free path planning across arbitrarily contoured terrain with anisotropic friction and gravity effects. IEEE Transactions on Robotics and Automation, 6(5):540-553, October 1990.

[8] Neil C. Rowe. Obtaining optimal mobile-robot paths with nonsmooth anisotropic cost functions using qualitative-state reasoning. The International Journal of Robotics Research, 16(3):375399, 1997.

[9] A. B. Philpott, R. M. Sullivan, and P. S. Jackson. Yacht velocity prediction using mathematical programming. European Journal of Operational Research,67(1):13-24, May 1993. 
[10] Toby Allsopp, Andrew Mason, and Andy Philpott. Optimal sailing routes with uncertain weather. In Proceedings of The 35th Annual Conference of the Operational Research Society of New Zealand, pages 65-74, December 2000.

[11] Andy B. Philpott and Andrew Mason. Optimising yacht routes under uncertainty. In The 15th Cheasapeake Sailing Yacht Symposium, 2001.

[12] A. B. Philpott. Stochastic optimization and yacht racing. In Applications of stochastic programming, volume 5 of MPS/SIAM Ser. Optim., pages 315-336. SIAM, Philadelphia, PA, 2005 .

[13] Jürgen Sellen. Direction weighted shortest path planning. In Proceedings of the International Conference on Robotics and Automation, pages 1970-1975. IEEE Computer Society, 1995.

[14] Frank D. Faulkner. A general numerical method for determining optimum ship routes. Navigation, 10(2):143-148, 1963.

[15] Frank D. Faulkner. Numerical methods for determining optimum ship routes. Navigation: Journal of The Institute of Navigation, 10(4):351-367, Winter 1963.

[16] Nikiforos A. Papadakis and Anastassios N. Perakis. Deterministic minimal time vessel routing. Oper. Res., 38(3):426-438, 1990.

[17] J. C. Kimball and H. Story. Fermat's principle, Huygens' principle, Hamilton's optics and sailing strategy. European Journal of Physics, 19:15-24, January 1998.

[18] John H. Reif and Zheng Sun. Movement planning in the presence of flows. Algorithmica, 39 (2):127-153, 2004.

[19] Siu-Wing Cheng, Hyeon-Suk Na, Antoine Vigneron, and Yajun Wang. Approximate shortest paths in anisotropic regions. SIAM J. Comput., 38(3):802-824, 2008.

[20] L. Paul Chew and Robert L. (Scot) Drysdale, III. Voronoi diagrams based on convex distance functions. In $S C G$ '85: Proceedings of the first annual symposium on Computational geometry, pages 235-244, New York, NY, USA, 1985. ACM. ISBN 0-89791-163-6.

[21] Tomás Lozano-Pérez and Michael A. Wesley. An algorithm for planning collision-free paths among polyhedral obstacles. Commun. ACM, 22(10):560-570, 1979.

[22] H. Alt and E. Welzl. Visibility graphs and obstacle-avoiding shortest paths. Z. Oper. Res., 32 (3-4):145-164, 1988.

[23] Irina S. Dolinskaya and Robert L. Smith. Path planning in an anisotropic medium. Technical Report 12-01, Northwestern University, available at http://www.iems.northwestern.edu/research/papers.html, 2012.

[24] David G. Luenberger. Optimization by vector space methods. John Wiley \& Sons Inc., New York, 1969. xvii+326 pp.

[25] J. W. S. Cassels. An introduction to the geometry of numbers. Die Grundlehren der mathematischen Wissenschaften in Einzeldarstellungen mit besonderer Berücksichtigung der Anwendungsgebiete, Bd. 99 Springer-Verlag, Berlin-Göttingen-Heidelberg, 1959. viii-344 pp. 
[26] Ronald L. Graham. An efficient algorithm for determining the convex hull of a finite planar set. Inf. Process. Lett., 1(4):132-133, 1972.

[27] Thomas H. Cormen, Charles E. Leiserson, Ronald L. Rivest, and Clifford Stein. Introduction to algorithms. MIT Press, Cambridge, MA, second edition, 2001. xxii-1180 pp. 